\title{
Chaos for Cosine Operator Functions on Groups
}

\author{
Chung-Chuan Chen \\ Department of Mathematics Education, National Taichung University of Education, Taichung 403, Taiwan \\ Correspondence should be addressed to Chung-Chuan Chen; chungchuan@mail.ntcu.edu.tw
}

Received 18 December 2013; Revised 11 February 2014; Accepted 11 February 2014; Published 13 March 2014

Academic Editor: Wei-Shih Du

Copyright (C) 2014 Chung-Chuan Chen. This is an open access article distributed under the Creative Commons Attribution License, which permits unrestricted use, distribution, and reproduction in any medium, provided the original work is properly cited.

Let $1 \leq p<\infty$ and $G$ be a locally compact group. We characterize chaotic cosine operator functions, generated by weighted translations on the Lebesgue space $L^{p}(G)$, in terms of the weight condition. In particular, chaotic cosine operator functions and chaotic weighted translations can only occur simultaneously. We also give a necessary and sufficient condition for the direct sum of a sequence of cosine operator functions to be chaotic.

\section{Introduction}

Let $T$ be a bounded linear operator on a Banach space $X$. If $v \in X$ is a fixed point of $T$, then the orbit of $v$ under $T$, denoted by $\operatorname{Orb}(T, v)$, is $\operatorname{Orb}(T, v)=\left\{v, T v, T^{2} v, \ldots\right\}=\{v\}$. On the contrary, if there exists an element $x \in X$ such that the orbit is dense in $X$; that is, $\overline{\operatorname{Orb}(T, x)}=\overline{\left\{x, T x, T^{2} x, \ldots\right\}}=X$, then $T$ is called hypercyclic and $x$ is a hypercyclic vector for $T$. Hypercyclicity arose from the invariant subset problem in analysis, and was studied intensely during the last two decades. We refer to $[1,2]$ for recent books on this subject.

In the study of hypercyclicity, the weighted shifts on $\ell^{p}(\mathbb{Z})$ play an important role for researchers to demonstrate and construct the theories in [3-11]. Recently, we characterize chaotic, hypercyclic, and mixing translation operators on locally compact groups in [12-15], which extends some results of weighted shifts on the discrete group $\mathbb{Z}$ in $[5,6,8-11]$ and provides a class of hypercyclic operators on Banach spaces. In this note, we will continue our study in $[16,17]$ and determine when a cosine operator function, generated by such a weighted translation operator, is chaotic.

Let $\mathbb{N}_{0}=\mathbb{N} \cup\{0\}$. According to the definition of Devaney chaos, a sequence of bounded linear operators $\left(T_{n}\right)_{n \in \mathbb{N}_{0}}$ on a Banach space $X$ is chaotic in the successive way in [18] if $\left(T_{n}\right)_{n \in \mathbb{N}_{0}}$ is topologically transitive and the set of periodic elements, denoted by $\mathscr{P}\left(\left(T_{n}\right)_{n \in \mathbb{N}_{0}}\right)=\{x \in X ; \exists m \in \mathbb{N}$ : $\left.T_{k m} x=x, k=1,2,3, \ldots\right\}$, is dense in $X$. We recall that $\left(T_{n}\right)_{n \in \mathbb{N}_{0}}$ is topologically transitive if, given nonempty open subsets $U, V$ of $X$, we have $T_{n}(U) \cap V \neq \emptyset$ for some $n \in$
$\mathbb{N}$. If $T_{n}(U) \cap V \neq \emptyset$ from some $n$ onwards, then $\left(T_{n}\right)_{n \in \mathbb{N}_{0}}$ is called topologically mixing. The notion of transitivity in topological dynamics is close to the notion of hypercyclicity in operator theory. Indeed, it is known in [19] that $\left(T_{n}\right)_{n \in \mathbb{N}_{0}}$ is transitive if, and only if, it is hypercyclic and has a dense set of hypercyclic vectors. In the more general setting, a sequence of operators $\left(T_{n}\right)_{n \in \mathbb{N}_{0}}$ is said to be hypercyclic if $\overline{\operatorname{Orb}\left(T_{n}, x\right)}=$ $\overline{\left\{T_{n} x: n \in \mathbb{N}_{0}\right\}}=X$ for some $x \in X$. If $\left(T_{n}\right)_{n \in \mathbb{N}_{0}}$ is generated by a single operator $T$ by its iterates, that is, $T_{n}:=T^{n}$, then hypercyclicity is equivalent to transitivity.

The interest to study cosine operator functions on groups is motivated by the work in [20,21]. A cosine operator function on a Banach space $X$ is a mapping $\mathscr{C}$ from the real line into the space of continuous operators on $X$ satisfying $\mathscr{C}(0)=I$ and the d'Alembert functional equation $2 \mathscr{C}(t) \mathscr{C}(s)=\mathscr{C}(t+$ $s)+\mathscr{C}(t-s)$ for all $s, t \in \mathbb{R}$, which implies $\mathscr{C}(t)=\mathscr{C}(-t)$ for all $t \in \mathbb{R}$. In [20], Bonilla and Miana obtained a sufficient condition for a cosine operator function $\mathscr{C}(t)$ defined by

$$
\mathscr{C}(t)=\frac{1}{2}(T(t)+T(-t)),
$$

to be transitive, where $T$ is a strongly continuous translation group on some weighted Lebesgue space $L^{p}(\mathbb{R})$. For a Borel measure $\mu$ and $\Omega \subset \mathbb{R}^{d}$, Kalmes gave the characterization for cosine operator functions, generated by second order partial differential operators on $L^{p}(\Omega, \mu)$, to be transitive and mixing in [21].

Throughout, let $G$ be a locally compact group with identity $e$. Let $\lambda$ be a right-invariant Haar measure on $G$, and 
denote by $L^{p}(G)(1 \leq p<\infty)$ the complex Lebesgue space with respect to $\lambda$.

A function $w: G \rightarrow(0, \infty)$ is called a weight on $G$. Let $a \in G$ and let $\delta_{a}$ be the unit point mass at $a$. A weighted translation on $G$ is a weighted convolution operator $T_{a, w}$ : $L^{p}(G) \rightarrow L^{p}(G)$ defined by

$$
T_{a, w}(f):=w T_{a}(f) \quad\left(f \in L^{p}(G)\right),
$$

where $w$ is a weight on $G$ and $T_{a}(f)=f * \delta_{a} \in L^{p}(G)$ is the convolution:

$$
\left(f * \delta_{a}\right)(x):=\int_{G} f\left(x y^{-1}\right) d \delta_{a}(y)=f\left(x a^{-1}\right) \quad(x \in G) .
$$

If $w^{-1} \in L^{\infty}(G)$, then the weighted translation operator $T_{a^{-1}, w^{-1} * \delta_{a}^{-1}}$ is the inverse of $T_{a, w}$. We write $S_{a, w}$ for $T_{a^{-1}, w^{-1} * \delta_{a}^{-1}}$ to simplify notation.

In what follows, we assume $w, w^{-1} \in L^{\infty}(G)$ and define a sequence of bounded linear operators $C_{n}: L^{p}(G) \rightarrow L^{p}(G)$ by

$$
C_{n}=\frac{1}{2}\left(T_{a, w}^{n}+S_{a, w}^{n}\right)
$$

for all $n \in \mathbb{Z}$ where $T_{a, w}^{-n}:=\left(T_{a, w}^{-1}\right)^{n}=S_{a, w}^{n}$. Then $\left(C_{n}\right)_{n \in \mathbb{Z}}$ can be regarded as a cosine operator function by letting $\mathscr{C}(n)=$ $C_{n}$. Since $C_{n}=C_{-n}$ for all $n \in \mathbb{Z}$, we will investigate the sequence of operators $\left(C_{n}\right)_{n \in \mathbb{N}_{0}}$ and give a necessary and sufficient condition for $\left(C_{n}\right)_{n \in \mathbb{N}_{0}}$ to be chaotic in terms of the weight function $w$, the Haar measure $\lambda$, and the group element $a \in G$.

\section{Chaotic Condition}

In this section, we will show the main result and give some examples of chaotic cosine operator functions on various groups. Since $\left(C_{n}\right)_{n \in \mathbb{N}_{0}}$ is generated by some element $a \in G$, we first note that $\left(C_{n}\right)_{n \in \mathbb{N}_{0}}$ is never chaotic if $a$ is a torsion element by the fact in [17] that $\left(C_{n}\right)_{n \in \mathbb{N}_{0}}$ is not transitive when $a$ is torsion.

Lemma 1. Let $G$ be a locally compact group and let a be a torsion element in $G$. Let $1 \leq p<\infty$ and $T_{a, w}$ be a weighted translation on $L^{p}(G)$ with inverse $S_{a, w}$. Let $C_{n}=(1 / 2)\left(T_{a, w}^{n}+\right.$ $\left.S_{a, w}^{n}\right)$. Then $\left(C_{n}\right)_{n \in \mathbb{N}_{0}}$ is not chaotic.

An element $a$ in a group $G$ is called a torsion element if it is of finite order. In a locally compact group $G$, an element $a \in G$ is called periodic [22] (or compact [23]) if the closed subgroup $G(a)$ generated by $a$ is compact. We call an element in $G$ aperiodic if it is not periodic. For discrete groups, periodic and torsion elements are identical; in other words, aperiodic elements are the nontorsion elements.

It has been shown in [15] that an element $a$ in a locally compact group $G$ is aperiodic if, and only if, for any compact subset $K \subset G$, there exists $N \in \mathbb{N}$ such that $K \cap K a^{ \pm n}=\emptyset$ for $n>N$. We will make use of the aperiodic condition to obtain the result.
Now we turn our attention to the set of periodic elements of $\left(C_{n}\right)_{n \in \mathbb{N}_{0}}$. Let $\mathscr{P}\left(\left(C_{n}\right)_{n \in \mathbb{N}_{0}}\right)$ be the set of periodic elements of a sequence of operator $\left(C_{n}\right)_{n \in \mathbb{N}_{0}}$. By the d'Alembert functional equation and induction, we have a simple observation immediately.

Lemma 2. Let $\mathscr{P}\left(\left(C_{n}\right)_{n \in \mathbb{N}_{0}}\right)$ be the set of periodic elements of $\left(C_{n}\right)_{n \in \mathbb{N}_{0}}$. Then $C_{m} f=f$ for some $m \in \mathbb{N}$ and $f \in \ell^{p}(\mathbb{Z})$ if, and only if, $f \in \mathscr{P}\left(\left(C_{n}\right)_{n \in \mathbb{N}_{0}}\right)$.

Proof. Let $C_{m} f=f$. By the d'Alembert functional equation, we have $C_{2 m} f=f$. Now assume $C_{k m} f=f$ and $C_{(k+1) m} f=$ $f$. Then, applying the d'Alembert functional equation again, we have

$$
\begin{aligned}
2 f & =2 C_{m} f=2 C_{m}\left(C_{(k+1) m} f\right) \\
& =C_{(k+2) m} f+C_{k m} f=C_{(k+2) m} f+f
\end{aligned}
$$

which says $C_{(k+2) m} f=f$.

Based on the work of characterizing transitive $\left(C_{n}\right)_{n \in \mathbb{N}_{0}}$ in [17], we are able to obtain the characterization for $\left(C_{n}\right)_{n \in \mathbb{N}_{0}}$ to be chaotic in this note. We state the result in [17] below.

Theorem 3 (see [17]). Let $G$ be a locally compact group and let $a$ be an aperiodic element in $G$. Let $1 \leq p<\infty$ and $T_{a, w}$ be a weighted translation on $L^{p}(G)$ with inverse $S_{a, w}$. Let $C_{n}=$ $(1 / 2)\left(T_{a, w}^{n}+S_{a, w}^{n}\right)$. Then the following conditions are equivalent.

(i) $\left(C_{n}\right)_{n \in \mathbb{N}_{0}}$ is topologically transitive.

(ii) For each compact subset $K \subset G$ with $\lambda(K)>0$, there are sequences of Borel sets $\left(E_{k}\right),\left(E_{k}^{+}\right)$, and $\left(E_{k}^{-}\right)$in $K$ such that $E_{k}=E_{k}^{+} \cup E_{k}^{-}, \lambda(K)=\lim _{k \rightarrow \infty} \lambda\left(E_{k}\right)$ and both sequences

$$
\varphi_{n}:=\prod_{s=1}^{n} w * \delta_{a^{-1}}^{s}, \quad \widetilde{\varphi}_{n}:=\left(\prod_{s=0}^{n-1} w * \delta_{a}^{s}\right)^{-1}
$$

admit, respectively, subsequences $\left(\varphi_{n_{k}}\right)$ and $\left(\widetilde{\varphi}_{n_{k}}\right)$ satisfying

$$
\begin{aligned}
& \lim _{k \rightarrow \infty}\left\|\left.\varphi_{n_{k}}\right|_{E_{k}}\right\|_{\infty}=\lim _{k \rightarrow \infty}\left\|\left.\widetilde{\varphi}_{n_{k}}\right|_{E_{k}}\right\|_{\infty}=0, \\
& \lim _{k \rightarrow \infty}\left\|\left.\varphi_{2 n_{k}}\right|_{E_{k}^{+}}\right\|_{\infty}=\lim _{k \rightarrow \infty}\left\|\left.\widetilde{\varphi}_{2 n_{k}}\right|_{E_{k}^{-}}\right\|_{\infty}=0 .
\end{aligned}
$$

Now we are ready to show the main result.

Theorem 4. Let $G$ be a locally compact group and let a be an aperiodic element in $G$. Let $1 \leq p<\infty$ and $T_{a, w}$ be a weighted translation on $L^{p}(G)$ with inverse $S_{a, w}$. Let $C_{n}=(1 / 2)\left(T_{a, w}^{n}+\right.$ $\left.S_{a, w}^{n}\right)$, and let $\mathscr{P}\left(\left(C_{n}\right)_{n \in \mathbb{N}_{0}}\right)$ be the set of periodic elements. Then the following conditions are equivalent.

(i) $\left(C_{n}\right)_{n \in \mathbb{N}_{0}}$ is chaotic.

(ii) $\mathscr{P}\left(\left(C_{n}\right)_{n \in \mathbb{N}_{0}}\right)$ is dense in $L^{p}(G)$. 
(iii) For each compact subset $K \subset G$ with $\lambda(K)>0$, there is a sequence of Borel sets $\left(E_{k}\right)$ in $K$ such that $\lambda(K)=$ $\lim _{k \rightarrow \infty} \lambda\left(E_{k}\right)$ and both sequences

$$
\varphi_{n}:=\prod_{s=1}^{n} w * \delta_{a^{-1}}^{s}, \quad \widetilde{\varphi}_{n}:=\left(\prod_{s=0}^{n-1} w * \delta_{a}^{s}\right)^{-1}
$$

admit, respectively, subsequences $\left(\varphi_{n_{k}}\right)$ and $\left(\widetilde{\varphi}_{n_{k}}\right)$ satisfying

$$
\lim _{k \rightarrow \infty}\left(\sum_{l=1}^{\infty} \int_{E_{k}} \varphi_{l n_{k}}^{p}(x) d \lambda(x)+\sum_{l=1}^{\infty} \int_{E_{k}} \widetilde{\varphi}_{l n_{k}}^{p}(x) d \lambda(x)\right)=0
$$

Proof. Since (i) $\Rightarrow$ (ii) is trivial, we only need to show (ii) $\Rightarrow$ (iii) and (iii) $\Rightarrow$ (i).

(ii) $\Rightarrow$ (iii). Let $\mathscr{P}\left(\left(C_{n}\right)_{n \in \mathbb{N}_{0}}\right)$ be dense in $L^{p}(G)$. Let $K$ be a compact subset of $G$ with $\lambda(K)>0$. Then by the aperiodicity, there exists some $N \in \mathbb{N}$ such that $K \cap K a^{ \pm m}=\emptyset$ for all $m>N$. Let $\chi_{K} \in L^{p}(G)$ be the characteristic function of $K$. By density of $\mathscr{P}\left(\left(C_{n}\right)_{n \in \mathbb{N}_{0}}\right)$, we can find a sequence $\left(n_{k}\right) \subset \mathbb{N}$ and a sequence $\left(f_{k}\right)$ of periodic points of $\left(C_{n}\right)_{n \in \mathbb{N}_{0}}$ such that $\left\|f_{k}-\chi_{K}\right\|_{p}<1 / 4^{k}$ and $C_{n_{k}} f_{k}=f_{k}$ in which we may assume $n_{k+1}>n_{k}>N$. Hence we have

$$
\frac{1}{4^{k}}>\left\|f_{k}-\chi_{K}\right\|_{p} \geq\left\|f_{k} \chi_{K}-\chi_{K}\right\|_{p}
$$

Let $A_{k}=\left\{x \in K:\left|f_{k} \chi_{K}(x)-1\right|>1 / 2^{k}\right\}$. Then we have

$$
\left|f_{k} \chi_{K}(x)\right| \geq 1-\frac{1}{2^{k}} \quad\left(x \in K \backslash A_{k}\right) .
$$

Also, by the inequality below

$$
\begin{aligned}
\frac{1}{4^{p k}} & >\left\|f_{k} \chi_{K}-\chi_{K}\right\|_{p}^{p} \\
& =\int_{G}\left|f_{k} \chi_{K}(x)-\chi_{K}(x)\right|^{p} d \lambda(x) \\
& \geq \int_{K}\left|f_{k} \chi_{K}(x)-1\right|^{p} d \lambda(x) \\
& \geq \int_{A_{k}}\left|f_{k} \chi_{K}(x)-1\right|^{p} d \lambda(x)>\frac{1}{2^{p k}} \lambda\left(A_{k}\right),
\end{aligned}
$$

we have $\lambda\left(A_{k}\right)<1 / 2^{p k}$. Hence $\lambda\left(K \backslash E_{k}\right)<1 / 2^{p k}$ by letting $E_{k}=K \backslash A_{k}$. Moreover, using $f_{k}=C_{l_{n}} f_{k}$ for $l \in \mathbb{N}$, the right invariance of the Haar measure $\lambda$ and $K a^{r n_{k}} \cap K a^{s n_{k}}=\emptyset$ for all $r, s \in \mathbb{Z}$ and $r \neq s$, we arrive at

$$
\begin{aligned}
& \frac{1}{4^{p k}}>\left\|f_{k} \chi_{K}-\chi_{K}\right\|_{p}^{p} \\
& =\int_{G}\left|f_{k} \chi_{K}(x)-\chi_{K}(x)\right|^{p} d \lambda(x) \\
& \geq \int_{G \backslash K}\left|f_{k} \chi_{K}(x)\right|^{p} d \lambda(x) \\
& \geq \sum_{l=1}^{\infty} \int_{K a^{l n_{k}}}\left|f_{k} \chi_{K}(x)\right|^{p} d \lambda(x) \\
& +\sum_{l=1}^{\infty} \int_{K a^{-l n_{k}}}\left|f_{k} \chi_{K}(x)\right|^{p} d \lambda(x) \\
& =\sum_{l=1}^{\infty} \int_{K}\left|f_{k} \chi_{K}\left(x a^{\ln _{k}}\right)\right|^{p} d \lambda(x) \\
& +\sum_{l=1}^{\infty} \int_{K}\left|f_{k} \chi_{K}\left(x a^{-\ln _{k}}\right)\right|^{p} d \lambda(x) \\
& =\frac{1}{2^{p}} \sum_{l=1}^{\infty} \int_{K}\left|T_{a, w}^{l n_{k}} f_{k} \chi_{K}\left(x a^{\ln _{k}}\right)+S_{a, w}^{\ln _{k}} f_{k} \chi_{K}\left(x a^{\ln _{k}}\right)\right|^{p} d \lambda(x) \\
& +\frac{1}{2^{p}} \sum_{l=1}^{\infty} \int_{K} \mid T_{a, w}^{l n_{k}} f_{k} \chi_{K}\left(x a^{-l n_{k}}\right) \\
& +\left.S_{a, w}^{l n_{k}} f_{k} \chi_{K}\left(x a^{-l n_{k}}\right)\right|^{p} d \lambda(x) \\
& \geq \frac{1}{2^{p}} \sum_{l=1}^{\infty} \int_{E_{k}} \mid \varphi_{l n_{k}}(x) f_{k} \chi_{K}(x) \\
& +\left.\varphi_{\ln _{k}}^{-1}\left(x a^{\ln _{k}}\right) f_{k} \chi_{K}\left(x a^{2 \ln _{k}}\right)\right|^{p} d \lambda(x) \\
& +\frac{1}{2^{p}} \sum_{l=1}^{\infty} \int_{E_{k}} \mid \widetilde{\varphi}_{\ln _{k}}^{-1}\left(x a^{-\ln _{k}}\right) f_{k} \chi_{K}\left(x a^{-2 \ln _{k}}\right) \\
& +\left.\widetilde{\varphi}_{l n_{k}}(x) f_{k} \chi_{K}(x)\right|^{p} d \lambda(x) \\
& =\frac{1}{2^{p}} \sum_{l=1}^{\infty} \int_{E_{k}}\left|\varphi_{l n_{k}}(x) f_{k} \chi_{K}(x)\right|^{p} d \lambda(x) \\
& +\frac{1}{2^{p}} \sum_{l=1}^{\infty} \int_{E_{k}}\left|\widetilde{\varphi}_{l n_{k}}(x) f_{k} \chi_{K}(x)\right|^{p} d \lambda(x) \\
& \geq \frac{1}{2^{p}}\left(1-\frac{1}{2^{k}}\right)^{p} \sum_{l=1}^{\infty} \int_{E_{k}} \varphi_{l n_{k}}^{p}(x) d \lambda(x) \\
& +\frac{1}{2^{p}}\left(1-\frac{1}{2^{k}}\right)^{p} \sum_{l=1}^{\infty} \int_{E_{k}} \widetilde{\varphi}_{l n_{k}}^{p}(x) d \lambda(x)
\end{aligned}
$$

which proves condition (iii).

(iii) $\Rightarrow$ (i). The proof is similar to the proof of $[12$, Theorem 2.1]. We include the argument for completeness. 
By Theorem 3, a sequence of operators $\left(C_{n}\right)_{n \in \mathbb{N}_{0}}$ is topologically transitive. Hence we will show $\mathscr{P}\left(\left(C_{n}\right)_{n \in \mathbb{N}_{0}}\right)$ is dense in $L^{p}(G)$. It is known that the space $C_{c}(G)$ of continuous functions on $G$ with compact support is dense in $L^{p}(G)$. Let $f \in C_{c}(G)$ with compact support $K \subset G$. There is a sequence of Borel sets $\left(E_{k}\right)$ in $K$ such that $\lambda(K)=\lim _{k \rightarrow \infty} \lambda\left(E_{k}\right)$ and

$$
\begin{aligned}
& \left\|T_{a, w}^{l n_{k}}\left(f \chi_{E_{k}}\right)\right\|_{p}^{p} \\
& =\int_{E_{k} a^{l n_{k}}}\left|w(x) w\left(x a^{-1}\right) \cdots w\left(x a^{-\left(\ln _{k}-1\right)}\right)\right|^{p} \\
& \quad \times\left|f\left(x a^{-l n_{k}}\right)\right|^{p} d \lambda(x) \\
& =\int_{E_{k}}\left|w\left(x a^{\ln _{k}}\right) w\left(x a^{l n_{k}-1}\right) \cdots w(x a)\right|^{p}|f(x)|^{p} d \lambda(x) \\
& =\int_{E_{k}} \varphi_{\ln _{k}}^{p}(x)|f(x)|^{p} d \lambda(x) \leq\|f\|_{\infty}^{p} \int_{E_{k}} \varphi_{\ln _{k}}^{p}(x) d \lambda(x) .
\end{aligned}
$$

Similarly,

$$
\left\|S_{a, w}^{l_{n_{k}}}\left(f \chi_{E_{k}}\right)\right\|_{p}^{p} \leq\|f\|_{\infty}^{p} \int_{E_{k}} \widetilde{\varphi}_{l_{n_{k}}}^{p}(x) d \lambda(x) .
$$

Let

$$
v_{k}=f \chi_{E_{k}}+\sum_{l=1}^{\infty} T_{a, w}^{l n_{k}}\left(f \chi_{E_{k}}\right)+\sum_{l=1}^{\infty} S_{a, w}^{l n_{k}}\left(f \chi_{E_{k}}\right)
$$

Then $v_{k} \in L^{p}(G)$ by the weight assumption in the condition (iii). Also, using $K a^{r n_{k}} \cap K a^{s n_{k}}=\emptyset$ again, we have $v_{k} \rightarrow f$ as $k \rightarrow \infty$ which follows from

$$
\begin{aligned}
& \left\|v_{k}-f\right\|_{p}^{p} \\
& \leq\|f\|_{\infty}^{p} \lambda\left(K \backslash E_{k}\right) \\
& +\sum_{l=1}^{\infty}\left\|T_{a, w}^{l n_{k}}\left(f \chi_{E_{k}}\right)\right\|_{p}^{p}+\sum_{l=1}^{\infty}\left\|S_{a, w}^{l n_{k}}\left(f \chi_{E_{k}}\right)\right\|_{p}^{p} \\
& \leq\|f\|_{\infty}^{p} \lambda\left(K \backslash E_{k}\right) \\
& +\|f\|_{\infty}^{p}\left(\sum_{l=1}^{\infty} \int_{E_{k}} \varphi_{l_{n_{k}}^{p}}(x) d \lambda(x)\right. \\
& \left.\quad+\sum_{l=1}^{\infty} \int_{E_{k}} \widetilde{\varphi}_{l_{l n}}^{p}(x) d \lambda(x)\right) .
\end{aligned}
$$

On the other hand, $v_{k}$ is an element of $\mathscr{P}\left(\left(C_{n}\right)_{n \in \mathbb{N}_{0}}\right)$ by the equality

$$
\begin{aligned}
2 C_{n_{k}} v_{k}= & T_{a, w}^{n_{k}}\left(f \chi_{E_{k}}\right)+\sum_{l=1}^{\infty} T_{a, w}^{n_{k}} T_{a, w}^{l n_{k}}\left(f \chi_{E_{k}}\right) \\
& +\sum_{l=1}^{\infty} T_{a, w}^{n_{k}} S_{a, w}^{n_{k}}\left(f \chi_{E_{k}}\right) \\
& +S_{a, w}^{n_{k}}\left(f \chi_{E_{k}}\right)+\sum_{l=1}^{\infty} S_{a, w}^{n_{k}} T_{a, w}^{l n_{k}}\left(f \chi_{E_{k}}\right) \\
& +\sum_{l=1}^{\infty} S_{a, w}^{n_{k}} S_{a, w}^{l n_{k}}\left(f \chi_{E_{k}}\right) \\
= & \sum_{l=1}^{\infty} T_{a, w}^{l n_{k}}\left(f \chi_{E_{k}}\right)+f \chi_{E_{k}}+\sum_{l=1}^{\infty} S_{a, w}^{l n_{k}}\left(f \chi_{E_{k}}\right) \\
& +f \chi_{E_{k}}+\sum_{l=1}^{\infty} T_{a, w}^{l n_{k}}\left(f \chi_{E_{k}}\right)+\sum_{l=1}^{\infty} S_{a, w}^{l n_{k}}\left(f \chi_{E_{k}}\right) \\
= & v_{k}+v_{k}=2 v_{k} .
\end{aligned}
$$

Putting all these together, condition (iii) implies (i).

We note that [15] in many familiar nondiscrete groups, including the additive group $\mathbb{R}^{d}$, the Heisenberg group, and the affine group, all elements except the identity are aperiodic. On the other hand, if $G$ is discrete, then $A_{k}=\emptyset$ and $E_{k}=K$ for all $k \in \mathbb{N}$ in the proof of Theorem 4 . Hence we have the characterization below for discrete groups.

Corollary 5. Let $G$ be a discrete group and let a be a nontorsion element in $G$. Let $1 \leq p<\infty$ and $T_{a, w}$ be a weighted translation on $\ell^{p}(G)$. Let $C_{n}=(1 / 2)\left(T_{a, w}^{n}+S_{a, w}^{n}\right)$. Then the following conditions are equivalent.

(i) $\left(C_{n}\right)_{n \in \mathbb{N}_{0}}$ is chaotic.

(ii) For each finite subset $K \subset G$, both sequences

$$
\varphi_{n}=\prod_{s=1}^{n} w * \delta_{a^{-1}}^{s}, \quad \tilde{\varphi}_{n}=\left(\prod_{s=0}^{n-1} w * \delta_{a}^{s}\right)^{-1}
$$

admit. Respectively, subsequences $\left(\varphi_{n_{k}}\right)$ and $\left(\widetilde{\varphi}_{n_{k}}\right)$ satisfying

$$
\lim _{k \rightarrow \infty}\left(\sum_{l=1}^{\infty} \sum_{K} \varphi_{l n_{k}}^{p}(x)+\sum_{l=1}^{\infty} \sum_{K} \widetilde{\varphi}_{l n_{k}}^{p}(x)\right)=0 .
$$

It is also interesting to know that condition (iii) in Theorem 4 is also the sufficient and necessary condition for $T_{a, w}$ to be chaotic in [12, Theorem 2.1]. In other words, $\left(C_{n}\right)_{n \in \mathbb{N}_{0}}$ is chaotic if, and only if, $T_{a, w}$ is chaotic. We conclude the result below.

Corollary 6. Let $G$ be a locally compact group and let a be an aperiodic element in $G$. Let $1 \leq p<\infty$ and $T_{a, w}$ be a weighted translation on $L^{p}(G)$ with inverse $S_{a, w}$. Let $C_{n}=(1 / 2)\left(T_{a, w}^{n}+\right.$ $\left.S_{a, w}^{n}\right)$. Then the following conditions are equivalent. 
(i) $\left(C_{n}\right)_{n \in \mathbb{N}_{0}}$ is chaotic.

(ii) $T_{a, w}$ is chaotic.

(iii) $S_{a, w}$ is chaotic.

(iv) $T_{a, w}^{n}$ is chaotic for all $n \in \mathbb{N}$.

(v) $S_{a, w}^{n}$ is chaotic for all $n \in \mathbb{N}$.

Proof. For $n \in \mathbb{N}$, we denote the set of periodic elements of the operator $T_{a, w}^{n}$ by $\mathscr{P}\left(T_{a, w}^{n}\right)=\left\{f \in L^{p}(G) ; \exists m \in \mathbb{N}\right.$ : $\left.\left(T_{a, w}^{n}\right)^{m} f=f\right\}$. We will show that conditions (ii) and (iii) are equivalent, and (ii) implies (iv).

(ii) $\Leftrightarrow$ (iii). It is known in [19] that an invertible operator is transitive, if and only if, its inverse is transitive. Also it is easy to see $\mathscr{P}\left(T_{a, w}\right)=\mathscr{P}\left(S_{a, w}\right)$. Hence we prove the equivalence.

(ii) $\Rightarrow$ (iv). By [24], $T_{a, w}^{n}$ is transitive for all $n \in \mathbb{N}$ if $T_{a, w}$ is transitive. Moreover, we note that $\mathscr{P}\left(T_{a, w}\right)=\mathscr{P}\left(T_{a, w}^{n}\right)$ for all $n \in \mathbb{N}$. Therefore condition (ii) implies (iv).

We end up this section with two examples on $G=\mathbb{Z}$ and $G=\mathbb{R}$, which says that one can construct many chaotic cosine operator functions on various groups.

Example 7. Let $G=\mathbb{Z}, a=1 \in \mathbb{Z}$ which is nontorsion. Let $w * \delta_{1}$ be a weight on $\mathbb{Z}$. Then the weighted translation operator $T_{1, w * \delta_{1}}$ on $\ell^{p}(\mathbb{Z})$ is the bilateral weighted forward shift $T$, studied in [11] and given by $T e_{j}=w_{j} e_{j+1}$ with $w_{j}=$ $w(j)$. Here $\left(e_{j}\right)_{j \in \mathbb{Z}}$ is the canonical basis of $\ell^{p}(\mathbb{Z})$ and $\left(w_{j}\right)_{j \in \mathbb{Z}}$ is a sequence of positive real numbers. Also, we have

$$
\begin{aligned}
& \varphi_{\text {ln }}(j)=\prod_{s=1}^{\ln }\left(w * \delta_{1}\right) * \delta_{-1}^{s}(j)=\prod_{s=0}^{\ln -1} w(j+s), \\
& \widetilde{\varphi}_{\ln }^{-1}(j)=\prod_{s=0}^{\ln -1}\left(w * \delta_{1}\right) * \delta_{1}^{s}(j)=\prod_{s=1}^{\ln } w(j-s) .
\end{aligned}
$$

Let $w^{-1} \in \ell^{\infty}(\mathbb{Z})$ and let $C_{n}=(1 / 2)\left(T^{n}+S^{n}\right)$ where $S$ is the inverse of $T=T_{1, w * \delta_{1}}$. Then by Corollary $5,\left(C_{n}\right)_{n \in \mathbb{N}_{0}}$ and $T_{1, w * \delta_{1}}$ are chaotic if, given $\varepsilon>0$ and $q \in \mathbb{N}$, there exists an arbitrarily large $n \in \mathbb{N}$ such that

$$
\sum_{l=1}^{\infty} \sum_{|j| \leq q}\left(\prod_{s=0}^{l n-1} w(j+s)\right)^{p}+\sum_{l=1}^{\infty} \sum_{|j| \leq q}\left(\frac{1}{\prod_{s=1}^{l n} w(j-s)}\right)^{p}<\varepsilon .
$$

In fact, there are many weight functions $w$ on $\mathbb{Z}$ satisfying the weight condition above. For example, one may define $w$ : $\mathbb{Z} \rightarrow(0, \infty)$ by

$$
w(j)= \begin{cases}\frac{1}{2} & \text { if } j \geq 0 \\ 2 & \text { if } j<0\end{cases}
$$

Example 8. Let $G=\mathbb{R}, a=2$, and $w$ be a weight on $\mathbb{R}$. Then the weighted translation $T_{2, w}$ on $L^{p}(\mathbb{R})$ is defined by

$$
\begin{gathered}
T_{2, w} f(x)=w(x) f(x-2) \quad\left(f \in L^{p}(\mathbb{R})\right), \\
\varphi_{\ln _{k}}(x)=\prod_{s=1}^{\ln _{k}} w * \delta_{-2}^{s}(x)=\prod_{s=1}^{\ln k} w(x+2 s), \\
\widetilde{\varphi}_{\ln _{k}}^{-1}(x)=\prod_{s=0}^{\ln _{k}-1} w * \delta_{2}^{s}(x)=\prod_{s=0}^{\ln _{k}-1} w(x-2 s) .
\end{gathered}
$$

Let $C_{n}=(1 / 2)\left(T_{2, w}^{n}+S_{2, w}^{n}\right)$ where $S_{2, w}=T_{2, w}^{-1}$. Then $\left(C_{n}\right)_{n \in \mathbb{N}_{0}}$ and $T_{2, w}$ are chaotic if, given a compact subset $K$ of $\mathbb{R}$, we have

$$
\begin{aligned}
\lim _{k \rightarrow \infty}\left(\sum_{l=1}^{\infty} \int_{K}\left(\prod_{s=1}^{\ln _{k}} w(x+2 s)\right)^{p} d \lambda(x)\right. \\
\left.\quad+\sum_{l=1}^{\infty} \int_{K}\left(\frac{1}{\prod_{s=0}^{l n_{k}-1} w(x-2 s)}\right)^{p} d \lambda(x)\right)=0 .
\end{aligned}
$$

Similarly, one may choose $w: \mathbb{R} \rightarrow(0, \infty)$ by

$$
w(x)= \begin{cases}\frac{1}{2} & \text { if } x \geq 1 \\ \frac{1}{2^{x}} & \text { if }-1<x<1 \\ 2 & \text { if } x \leq-1,\end{cases}
$$

which is the required weight function in the above condition.

\section{The Direct Sum of Cosine Operator Functions}

Following the investigation on transitivity of the direct sum of a sequence of cosine operator functions in [17], we will give, in this section, the characterization for the direct sum of a sequence of cosine operator functions to be chaotic in terms of the similar weight condition in Theorem 4 . The work on the direct sum of operators in linear dynamics has been studied by many authors, for example $[5,9,11]$, where the notion of transitivity on direct sum of operators is related with another notion, namely, weak mixing, and hypercyclic criterion.

Given some $N \in \mathbb{N}$, let $\left(T_{a_{m}, w_{m}}\right)$ be a sequence of weighted translation operators on $L^{p}(G)$, defined by sequences of aperiodic elements $\left(a_{m}\right)$ in $G$ and positive weight functions $\left(w_{m}\right)$ for $1 \leq m \leq N$. We write $T_{m}$ for $T_{a_{m}, w_{m}}$ for simplification. In [17], we have the result below.

Corollary 9 (see [17]). Let $T_{m}$ be a weighted translation defined above for $1 \leq m \leq N$. Let $C_{m, n}=(1 / 2)\left(T_{m}^{n}+S_{m}^{n}\right)$. Then the following conditions are equivalent.

(i) $\left(C_{1, n} \oplus C_{2, n} \oplus \cdots \oplus C_{N, n}\right)_{n \in \mathbb{N}_{0}}$ is topologically transitive.

(ii) For $1 \leq m \leq N$ and each compact subset $K \subset G$ with $\lambda(K)>0$, there are sequences of Borel sets $\left(E_{m, k}\right)$, 
$\left(E_{m, k}^{+}\right)$, and $\left(E_{m, k}^{-}\right)$in $K$ such that $E_{m, k}=E_{m, k}^{+} \cup E_{m, k}^{-}$, $\lambda(K)=\lim _{k \rightarrow \infty} \lambda\left(E_{m, k}\right)$ and both sequences

$$
\varphi_{m, n}:=\prod_{s=1}^{n} w_{m} * \delta_{a_{m}^{-1}}^{s}, \quad \widetilde{\varphi}_{m, n}:=\left(\prod_{s=0}^{n-1} w_{m} * \delta_{a_{m}}^{s}\right)^{-1}
$$

admit, respectively, subsequences $\left(\varphi_{m, n_{k}}\right)$ and $\left(\widetilde{\varphi}_{m, n_{k}}\right)$ satisfying

$$
\begin{aligned}
& \lim _{k \rightarrow \infty}\left\|\left.\varphi_{m, n_{k}}\right|_{E_{m, k}}\right\|_{\infty}=\lim _{k \rightarrow \infty}\left\|\left.\tilde{\varphi}_{m, n_{k}}\right|_{E_{m, k}}\right\|_{\infty}=0, \\
& \lim _{k \rightarrow \infty}\left\|\left.\varphi_{m, 2 n_{k}}\right|_{E_{m, k}^{+}}\right\|_{\infty}=\lim _{k \rightarrow \infty}\left\|\left.\widetilde{\varphi}_{m, 2 n_{k}}\right|_{E_{m, k}^{-}}\right\|_{\infty}=0 .
\end{aligned}
$$

Using Corollary above, we characterize chaos of the direct sum of a sequence of cosine operator functions below.

Corollary 10. Let $T_{m}$ be a weighted translation for $1 \leq m \leq N$. Let $C_{m, n}=(1 / 2)\left(T_{m}^{n}+S_{m}^{n}\right)$. Then the following conditions are equivalent.

(i) $\left(C_{1, n} \oplus C_{2, n} \oplus \cdots \oplus C_{N, n}\right)_{n \in \mathbb{N}_{0}}$ is chaotic.

(ii) For $1 \leq m \leq N$ and each compact subset $K \subset G$ with $\lambda(K)>0$, there are sequences of Borel sets $\left(E_{m, k}\right)$ in $K$ such that $\lambda(K)=\lim _{k \rightarrow \infty} \lambda\left(E_{m, k}\right)$ and both sequences

$$
\varphi_{m, n}:=\prod_{s=1}^{n} w_{m} * \delta_{a_{m}^{-1}}^{s}, \quad \widetilde{\varphi}_{m, n}:=\left(\prod_{s=0}^{n-1} w_{m} * \delta_{a_{m}}^{s}\right)^{-1}
$$

admit, respectively, subsequences $\left(\varphi_{m, n_{k}}\right)$ and $\left(\widetilde{\varphi}_{m, n_{k}}\right)$ satisfying

$$
\begin{aligned}
\lim _{k \rightarrow \infty}\left(\sum_{l=1}^{\infty} \int_{E_{m, k}} \varphi_{m, l n_{k}}^{p}(x) d \lambda(x)\right. \\
\left.\quad+\sum_{l=1}^{\infty} \int_{E_{m, k}} \widetilde{\varphi}_{m, l n_{k}}^{p}(x) d \lambda(x)\right)=0 .
\end{aligned}
$$

Proof. The proof is similar to the proof of Theorem 4 .

(i) $\Rightarrow$ (ii). By density of $\mathscr{P}\left(\left(C_{m, n}\right)_{n \in \mathbb{N}_{0}}\right)$, we can find a sequence $\left(n_{k}\right) \subset \mathbb{N}$ and sequences $\left(f_{m, k}\right)$ of periodic points of $\left(C_{m, n}\right)_{n \in \mathbb{N}_{0}}$ such that $\left\|f_{m, k}-\chi_{K}\right\|_{p}<1 / 4^{k}$ and $C_{m, n_{k}} f_{m, k}=$ $f_{m, k}$. Let $E_{m, k}=K \backslash A_{m, k}$ for each $m$. Following the same estimation in the proof of Theorem 4 , we can obtain the weight conditions.

(ii) $\Rightarrow$ (i). Let $f_{m} \in C_{c}(G)$ with compact support $K_{m} \subset G$ for $1 \leq m \leq N$, and let $K=\cup_{m=1}^{N} K_{m}$. Letting

$$
\begin{aligned}
v_{m, k}:= & f_{m} \chi_{E_{m, k}}+\sum_{l=1}^{\infty} T_{m}^{l n_{k}}\left(f_{m} \chi_{E_{m, k}}\right) \\
& +\sum_{l=1}^{\infty} S_{m}^{l n_{k}}\left(f_{m} \chi_{E_{m, k}}\right) .
\end{aligned}
$$

and repeating the argument in the proof of Theorem 4, one can show that $v_{m, k} \rightarrow f_{m}$ as $k \rightarrow \infty$ and $v_{m, k}$ is an element of $\mathscr{P}\left(\left(C_{m, n}\right)_{n \in \mathbb{N}_{0}}\right)$. Hence $\left(C_{1, n} \oplus C_{2, n} \oplus \cdots \oplus C_{N, n}\right)_{n \in \mathbb{N}_{0}}$ is chaotic.
Remark 11. By Corollary 10 above, we note that $\left(C_{n}\right)_{n \in \mathbb{N}_{0}}$ is chaotic if, and only if, $\left(C_{n} \oplus C_{n}\right)_{n \in \mathbb{N}_{0}}$ is chaotic. Similarly, $T_{a, w}$ and $T_{a, w} \oplus T_{a, w}$ can be chaotic only at the same time.

Finally, we give an example of the direct sum of cosine operator functions on $\ell^{p}(\mathbb{Z})$.

Example 12. Given some $N \in \mathbb{N}$, let $G=\mathbb{Z}$ and $a_{m}=1$ for $1 \leq$ $m \leq N$. We consider a sequence of weighted translations $\left(T_{m}\right)$ on $\ell^{2}(\mathbb{Z})$, given by $T_{m}:=T_{1, w_{m} * \delta_{1}}$ where $\left(w_{m}\right)$ is a sequence of positive weight functions. Then we define sequences of operators $\left(C_{m, n}\right)_{n \in \mathbb{N}_{0}}$ by $C_{m, n}=(1 / 2)\left(T_{m}^{n}+S_{m}^{n}\right)$ where $S_{m}$ is the inverse of $T_{m}$. By Corollary 10, $\left(C_{1, n} \oplus C_{2, n} \oplus \cdots \oplus C_{N, n}\right)_{n \in \mathbb{N}_{0}}$ is chaotic if, given $\varepsilon>0$ and $q \in \mathbb{N}$, there exists an arbitrarily large $n$ such that for all $|j| \leq q$ and $1 \leq m \leq N$, we have

$$
\begin{aligned}
\sum_{l=1}^{\infty} \sum_{|j| \leq q}\left(\prod_{s=0}^{l n-1} w_{m}(j+s)\right)^{p} & )^{p}<\varepsilon .
\end{aligned}
$$

\section{Conflict of Interests}

The author declares that there is no conflict of interests regarding the publication of this paper.

\section{Acknowledgments}

The author would like to thank the reviewers for numerous helpful suggestions and remarks on this paper. The author is supported by NSC of Taiwan under Grant no. NSC 102-2115M-142-001-.

\section{References}

[1] F. Bayart and E. Matheron, Dynamics of Linear Operators, Cambridge Tracts in Math. 179, Cambridge University Press, Cambridge, Mass, USA, 2009.

[2] K.-G. Grosse-Erdmann and A. Peris, Linear Chaos, Universitext, Springer, 2011.

[3] C. Badea, S. Grivaux, and V. Müller, "Multiples of hypercyclic operators," Proceedings of the American Mathematical Society, vol. 137, no. 4, pp. 1397-1403, 2009.

[4] F. Bayart and S. Grivaux, "Frequently hypercyclic operators," Transactions of the American Mathematical Society, vol. 358, no. 11, pp. 5083-5117, 2006.

[5] J. Bès and A. Peris, "Hereditarily hypercyclic operators," Journal of Functional Analysis, vol. 167, no. 1, pp. 94-112, 1999.

[6] G. Costakis and M. Sambarino, "Topologically mixing hypercyclic operators," Proceedings of the American Mathematical Society, vol. 132, no. 2, pp. 385-389, 2004.

[7] R. Delaubenfels and H. Emamirad, "Chaos for functions of discrete and continuous weighted shift operators," Ergodic Theory and Dynamical Systems, vol. 21, no. 5, pp. 1411-1427, 2001.

[8] K.-G. Grosse-Erdmann, "Hypercyclic and chaotic weighted shifts," Studia Mathematica, vol. 139, no. 1, pp. 47-68, 2000. 
[9] F. León-Saavedra, "Operators with hypercyclic Cesàro means," Studia Mathematica, vol. 152, no. 3, pp. 201-215, 2002.

[10] A. Montes-Rodríguez and H. N. Salas, "Supercyclic subspaces: spectral theory and weighted shifts," Advances in Mathematics, vol. 163, no. 1, pp. 74-134, 2001.

[11] H. Salas, "Hypercyclic weighted shifts," Transactions of the American Mathematical Society, vol. 347, pp. 993-1004, 1995.

[12] C.-C. Chen, "Chaotic weighted translations on groups," Archiv der Mathematik, vol. 97, no. 1, pp. 61-68, 2011.

[13] C.-C. Chen, "Supercyclic and Cesàro hypercyclic weighted translations on groups," Taiwanese Journal of Mathematics, vol. 16, pp. 1815-1827, 2012.

[14] C.-C. Chen, "Hypercyclic weighted translations generated by non-torsion elements," Archiv der Mathematik, vol. 101, no. 2, pp. 135-141, 2013.

[15] C. Chen and C.-H. Chu, "Hypercyclic weighted translations on groups," Proceedings of the American Mathematical Society, vol. 139, no. 8, pp. 2839-2846, 2011.

[16] S.-J. Chang and C.-C. Chen, "Topological mixing for cosine operator functions generated by shifts," Topology and its Applications, vol. 160, no. 2, pp. 382-386, 2013.

[17] C.-C. Chen, Topological transitivity for cosine operator functions on groups.

[18] C. Tian and G. Chen, "Chaos of a sequence of maps in a metric space," Chaos, Solitons and Fractals, vol. 28, no. 4, pp. 1067-1075, 2006.

[19] K.-G. Karl-Goswin and G.-E. Grosse-Erdmann, "Universal families and hypercyclic operators," Bulletin of the American Mathematical Society, vol. 36, no. 3, pp. 345-381, 1999.

[20] A. Bonilla and P. J. Miana, "Hypercyclic and topologically mixing cosine functions on banach spaces," Proceedings of the American Mathematical Society, vol. 136, no. 2, pp. 519-528, 2008.

[21] T. Kalmes, "Hypercyclicity and mixing for cosine operator functions generated by second order partial differential operators," Journal of Mathematical Analysis and Applications, vol. 365, no. 1, pp. 363-375, 2010.

[22] S. Grosser and M. Moskowitz, "On central topological groups," Transactions of the American Mathematical Society, vol. 127, pp. 317-340, 1967.

[23] E. Hewitt and K. A. Ross, Abstract Harmonic Analysis, Springer, Heidelberg, Germany, 1979.

[24] S. I. Ansari, "Hypercyclic and Cyclic Vectors," Journal of Functional Analysis, vol. 128, no. 2, pp. 374-383, 1995. 


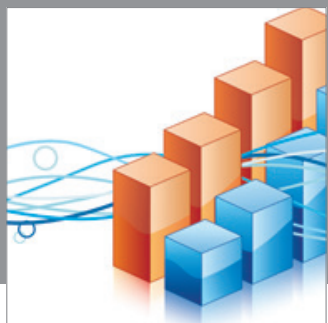

Advances in

Operations Research

mansans

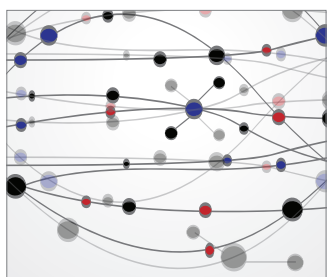

The Scientific World Journal
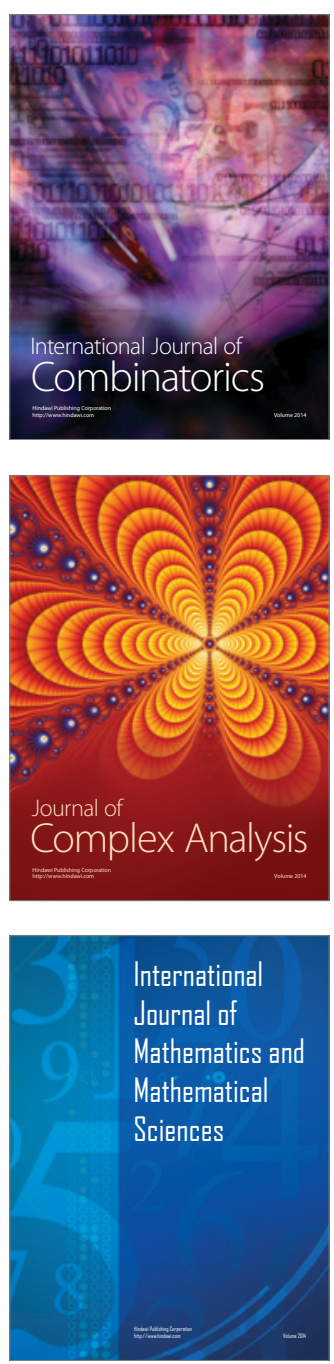
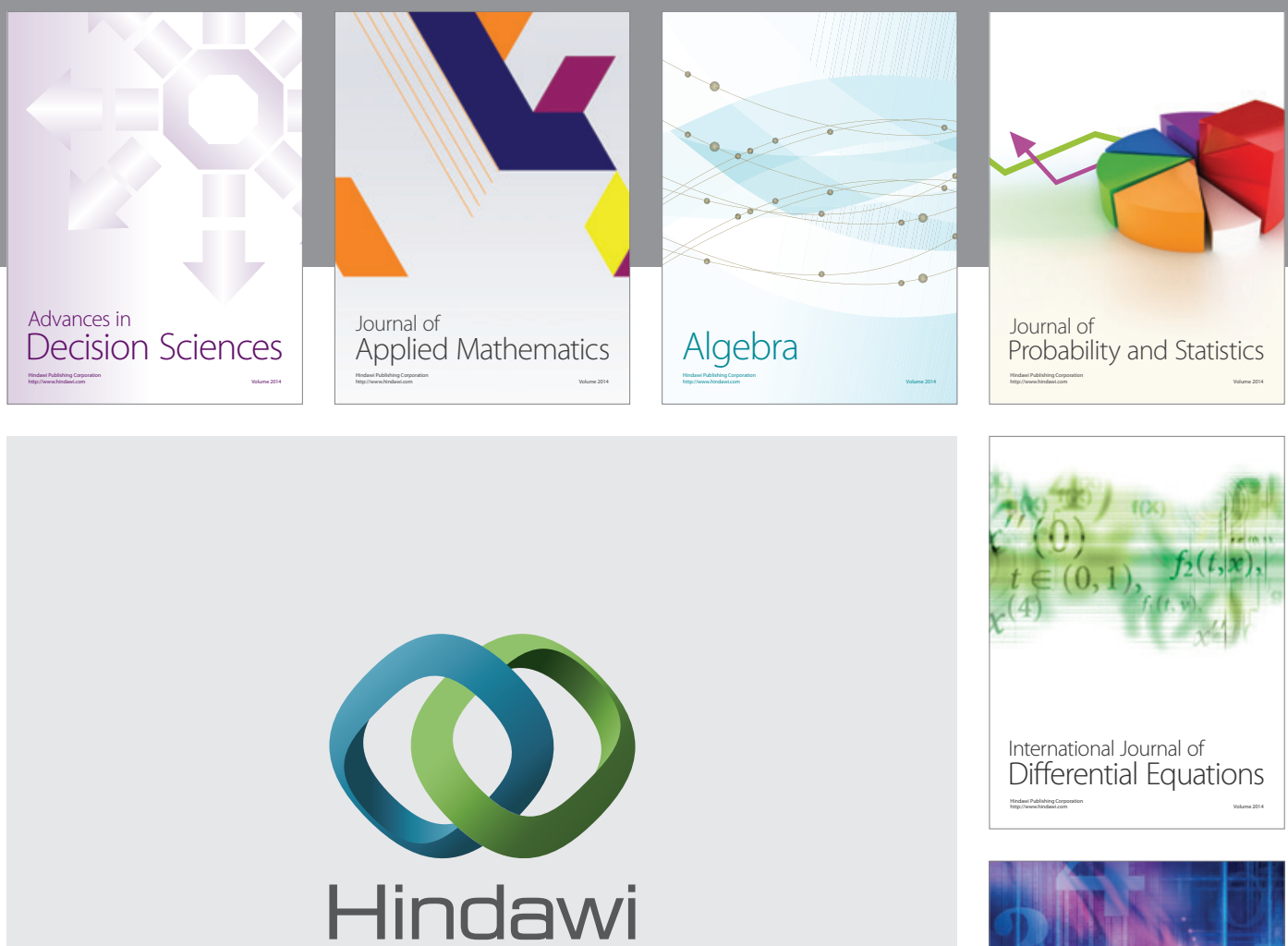

Submit your manuscripts at http://www.hindawi.com
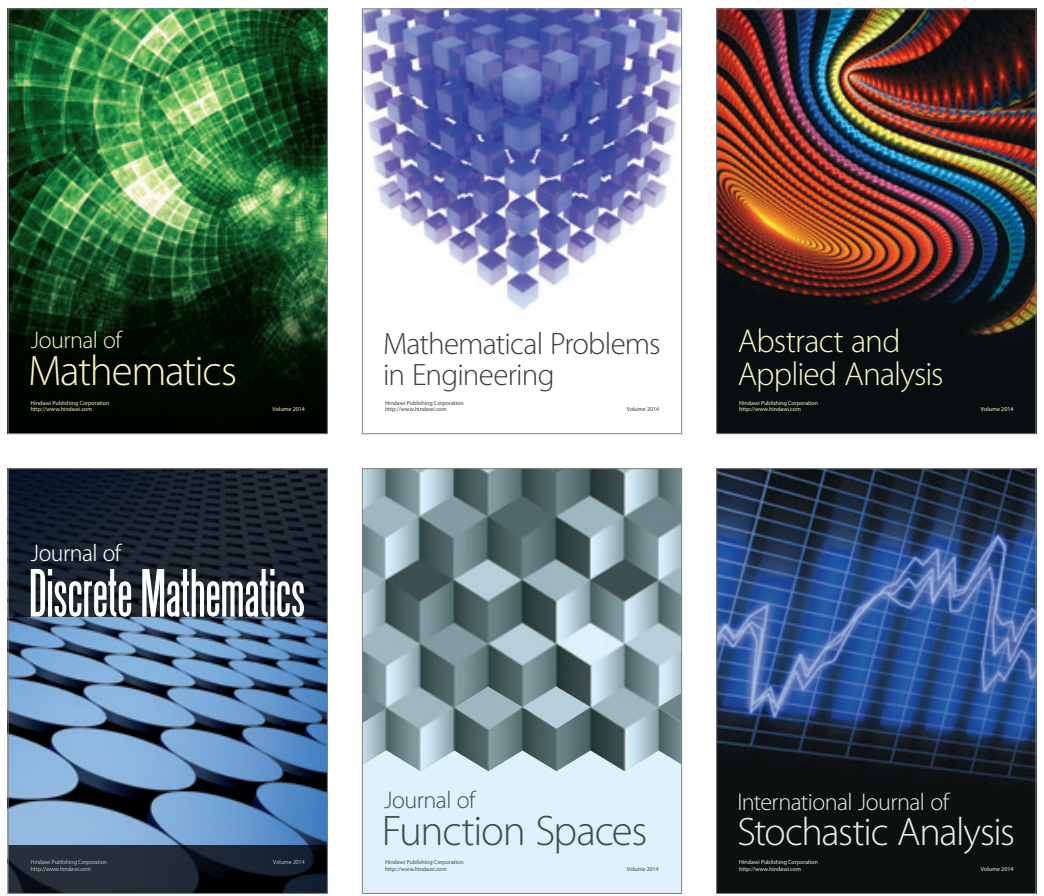

Journal of

Function Spaces

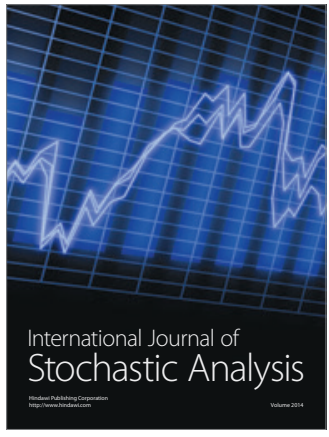

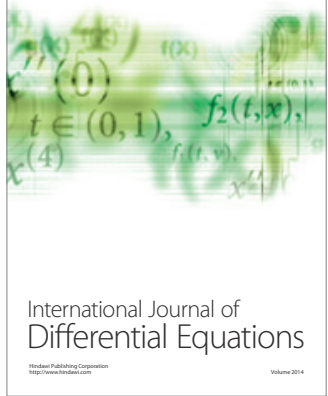
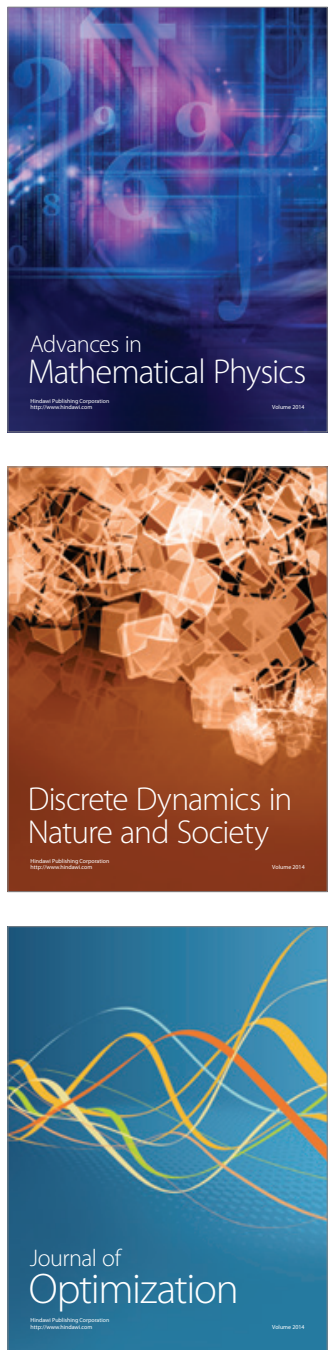